\title{
Use of Ionizing Radiation in the Health Sector
}

\author{
Katia Soares da Poça ${ }^{1 *}$, Luiz Antonio da Costa Rodrigues ${ }^{1}$ and Lilian Calazans Costa ${ }^{2}$ \\ ${ }^{1}$ School of Health, Celso Lisboa Center University, Rio de Janeiro, Brazil \\ ${ }^{2}$ Life and Health Sciences Research Institute (ICVS), School of Medicine, University of Minho, Portugal
}

*Corresponding author: Katia Soares da Poça, Sala dos Professores / Escola de Saúde. Centro Universitário Celso Lisboa. Rua Vinte

e Quatro de Maio, 797 - Engenho Novo, Rio de Janeiro/RJ, Brasil. CEP 20950-092

ARTICLE INFO

Received: 蔧 August 13, 2020

Published: 㹃 September 09, 2020

Citation: Katia Soares da Poça, Luiz Antonio da Costa Rodrigues, Lilian Calazans Costa. Use of Ionizing Radiation in the Health Sector. Biomed J Sci \& Tech Res 30(2)-2020. BJSTR. MS.ID.004920.

Keywords: Radiation, Ionizing; Health Personnel; Occupational Exposure; Cancer; Communication; Education; Public Health

Abbreviations: CNS: Central Nervous System; CNEN: National Nuclear Energy Commission; ICU: Intensive Care Units; IR: Ionizing Radiation

\section{ABSTRACT}

Ionizing Radiation (IR) is widely used in different applications. The energy capable of moving electrons in some materials has been used in different human activities, including in the health sector. Technological advances and diversity in the use of IR are expressed in the treatment of tumors, in diagnostic imaging and in the monitoring of invasive procedures. The performance of procedures with application of IR requires structural adequacy and procedures to guarantee the radiological protection of the patient and the source operator. The present work sought to carry out a narrative review of the literature on ionizing radiation, with the inclusion of definitions and concepts and the presentation of theories and evidence that helped to emphasize the topic of interest. International initiatives have been pioneering in the investigation of security and dosimetry parameters, however, there are still technical and conceptual gaps that do not allow the full implementation of international standards. The radioprotection theme needs to be widely discussed so that professionals who are currently exposed, even without operating radioactive sources, have access to adequate care procedures and guarantees. Continuing educational actions represent an important strategy for reducing health problems, including cancer, and improving workers' quality of life.

\section{Introduction}

The displacement of energy by electromagnetic waves or particles can vary in intensity and have different relationships with the propagation medium and have natural or anthropogenic sources. In the case of ionizing radiation, the high level of energy originated from the nucleus can change the state of an atom causing the loss of electrons, characterizing the ionization process, that is, the ability to become electrically charged due to the loss of electrons [1]. Normally, the radiation energy emitted during the nuclear transformation is measured in electron-volts $(\mathrm{eV})$, which represents a small radiation unit. Ionizing radiation normally has its energy represented by kilo $\mathrm{eV}(\mathrm{KeV}=1.000 \mathrm{eV})$ or mega $\mathrm{eV}(\mathrm{MeV}$ $\left.=10^{6} \mathrm{eV}\right)$ [2]. Unstable nuclei are capable of emitting particles $(\alpha$, $\beta$ or neutrons) and energy through electromagnetic waves ( $\gamma$-rays, which are similar to X-rays), in a process called ionization. This process allows the natural production of ionizing radiation of the $\alpha$, $\beta$ and $\gamma$ types. [1]. While the $\gamma$-ray is the only type of electromagnetic wave originating from the nucleus of unstable atoms; X-rays are usually photons emitted by the release of electrons that are distributed around an atom nucleus. However, X-rays and $\gamma$ have the ability to pass through different materials, such as paper, aluminum and lead due to the extremely high speed achieved, being therefore extremely penetrating and stopped only by concrete barriers $[1,2]$. The wavelengths of these types of ionizing radiation overlap, so that while X-rays reach energies with a few dozen $\mathrm{eV}, \gamma$-rays reach a few dozen $\mathrm{MeV}$ [1].

The earth's surface promotes natural exposure to ionizing radiation of types $\mathrm{X}$ and $\gamma$, since the earth's crust allows radon gas to emanate, allowing its presence in the soil, water, food and air $[3,4]$. Radon exposure is the main natural source of ionizing radiation. In the world, it is estimated that $40 \%$ of all exposure to this type of radiation, in the human population, is due to radon [1]. However, radiation with energy capable of displacing electrons and consequently producing ions is widely applied in industry and hospitals. In the health area, the use of ionizing radiation 
has brought many benefits, such as its application in diagnostic tests and in different therapies [2,5], due to the great capacity of penetration in different surfaces.

X-rays can be produced by an artificial source that accelerates electrons, followed by their collision against a lead plate. During the collision, there is a loss of kinetic energy by the electrons, causing heat production (most of the ionization process) and X-rays. From artificial mechanisms for the production of ionizing radiation, $\mathrm{X}$-rays, $\gamma$-rays, as well as radioactive particles (isotopes) can be used in health as: (1) Radiotherapy - use of X-rays, $\gamma$ and beams of electrons to treat tumors by eliminating tumor cells and consequent growth inhibition; (2) Brachytherapy - localized use of radiotherapy for specific types and locations of tumors, in which radioactive sources emitting low and medium energy gamma radiation are used; (3) Medicines containing radioisotopes - use of radioactive particles with guaranteed preferential deposition in target organs and which are administered orally or injectable to patients with tumors, such as the use of iodine isotopes for the treatment of thyroid cancer. Diagnostic exams, such as radiographs (use of X-rays or $\gamma$-rays), computed tomography (use of X-rays), mammography (use of low-energy X-ray beam) and mapping with radiopharmaceuticals (use of radioactive markers) are also widely performed in the health field [1].

The use of ionizing radiation in health services applies external and internal sources. While the external source is related to exposure to X-ray equipment [2], for example, the internal sources are related to radiopharmaceutical therapy [1], with the consumption of contaminated water and / or food or even dermal or respiratory contact with radionucleotides. According to the International Agency for Research on Cancer (IARC) all types of ionizing radiation are considered carcinogenic to humans and therefore classified in group I [2]. The absorption of ionizing radiation by the body contributes to the production of free radicals [4]. According to José Alencar Gomes da Silva National Cancer Institute (INCA), the main acute effects on the human species, due to exposure for a short period of time, usually to high doses, are nausea, weakness, hair loss, skin burns or decreased organic function. In general, cancer patients and those treated with radiation often experience these types of side effects to ongoing treatment [6]. However, in cases of accidents, for example, exposure can occur to very high doses of radiation, capable of causing damage so intense that it can contribute to death [4].

Exposure to small doses of radiation, over time, also contribute to human illness. The mechanism of action involved is the production of changes in DNA (initiation step with epigenetic mechanisms participation) that contribute to the development of different neoplasms such as cancer of the salivary gland, esophagus, stomach, colon, lung, bones, breast, bladder, kidney, skin, brain and Central Nervous System (CNS), thyroid and leukemia. These types of cancer are described in the literature due to chronic exposure to X-rays and $\gamma$-rays $[2,6]$. Despite the benefits, ionizing radiation exposes multidisciplinary health teams to the associated risks. In this way, workers from medical institutions and or laboratories who perform their occupational activities operating equipment that emit radiation are exposed to these carcinogenic agents [6]. In Brazil, the National Nuclear Energy Commission limits occupational exposure to an average of 20 millisievert ( $\mathrm{mSv}$ ) over a period of 5 years, but in one year, this exposure cannot exceed $50 \mathrm{mSv}$ [7], with the intention of minimizing risk to workers' health. The risk for the development of cancer depends on the dose, duration of exposure, age at which the exposure took place and tissue sensitivity to the carcinogenic effects of radiation [6].

In Latin America, tests involving ionizing radiation are performed by nurses, doctors and radiology technicians, but only $64 \%$ of the 10 largest cardiac intervention centers in Latin America use dosimeters. Of these, only $36 \%$ know how to interpret the results [8]. The lack of knowledge about the risks of radiation is also evident in Intensive Care Units (ICU). In situations in which the patient cannot move, the equipment is taken to the bed and the examinations are carried out without the adoption of adequate protection measures [9], putting everyone in the environment at risk. Clinical and epidemiological studies show deleterious effects on human health such as mutations, cancer, congenital abnormalities, cardio and cerebrovascular diseases, cataracts and others, even when exposed to low doses of ionizing radiation [10]. Exposure for prolonged periods, the absence of isolation measures similar to those adopted for equipment operators that emit ionizing radiation and the inappropriate use of individual and collective protection equipment [11], can constitute an occupational risk for a multidisciplinary team. Thus, the present work sought to carry out a narrative review of the literature on ionizing radiation, with the inclusion of definitions and concepts and the presentation of theories and evidence that helped to problematize the topic of interest. The inclusion criteria were articles published in Portuguese and English, available in the Pubmed, Science direct and Medline databases, with a focus on promoting knowledge for occupational health of the multidisciplinary health team. All relevant publications were considered, regardless of the date of publication, but those that were not available in full were excluded. Searches were also used in normative documents from the National Nuclear Energy Commission (CNEN), Oswaldo Cruz Foundation (FIOCRUZ), José Alencar Gomes da Silva National Cancer Institute (INCA), International Agency for Research on Cancer (IARC) and World Health Organization (WHO). The health descriptors (DeCs) used were 'Radiation, Ionizing', 'Occupational Exposure', 'Cancer' and 'Health Personnel'.

\section{Discussion}

$\mathrm{X}$-rays are widely used in clinical diagnosis and can be artificially generated by equipment (sources) that can be fixed or mobile and 
capable of inducing ionization of atoms [4]. The ability to react with different materials allows its application in the sterilization of materials, in the treatment of tumors and in diagnostic imaging, representing a huge advance in the treatment and prognosis of different pathologies. The use occurs from x-ray, computed tomography and radiotherapy devices [1], allowing the detection of anatomophysiological anomalies, injuries and verification of catheter fixation. The use of sources of ionizing radiation in its different forms of presentation presents a risk to the operator, justifying a series of occupational protection measures. In Brazil, regulatory standard No. 32, item 4 (NR-32.4), specifically addresses occupational exposure to ionizing radiation [11]. Item 32.4 .3 of the Brazilian standard is specific to workers and informs that everyone who works in areas with a source of ionizing radiation must: remain in these areas for the shortest time possible when performing the procedure; in addition to knowledge about the risks associated with radiological exposure, they need to be in continuous training in radiological protection; make use of personal protective equipment, in addition to being under individual monitoring of the dose of ionizing radiation [11].

In hospital facilities whose space for hospitalization of patients is reduced, there may be an increase in the exposure of professionals in the sector, in addition to exposure to unqualified professionals and patients. In this respect, the multidisciplinary team that assists patients in inpatient units, specifically those in intensive care, ends up being exposed due to the performance of radiological exams in the patient's bed. Although mobile devices have a configuration for safe operation in inpatient units $[7,11]$, the dimensioning and adaptation of environments can represent risk to the worker and the patient. In this context, epidemiological studies with populations exposed to radiotherapy, show an increased risk for the development of cancer, when the exposure occurs above $100 \mathrm{mSv}$ [4]. Exposures between 50 and $100 \mathrm{mSv}$ also showed a risk for children hospitalized in ICUs for cancer control [4]. With regard to epidemiological studies with workers in the health sector, these are scarce, whether these studies with operators of a source of ionizing radiation, or even with a multidisciplinary health team As the application of ionizing radiation in health increases every day, the continuous planning and development of actions to be adopted in different situations of exposure to ionizing radiation is extremely urgent, considering the risks involved in human health, but also in the environment. These measures gain importance when reviewing national experiences, such as the Cesium-137 accident, and international ones, realizing that the technical and logistical capacity of organizations, cities, states and countries may not be sufficient without prior planning [5]. The perception of the need to expand radioprotection measures was the target of international investigations in the search for standardization of acceptable parameters and protection measures. Although the movement in the search for standardization of protection measures has generated training courses and the establishment of collaboration networks, it is believed that it is necessary to expand the discussion and regulation. Hankin \& Jones (2020) [12] evaluated the impact of educational intervention on radiation protection in health professionals at different levels and realized that the intervention was effective in increasing knowledge on the topic, but found great variability of knowledge among participants in addition to recognize the absence of studies that assess the impact of this knowledge in the long term [12].

Thus, the efforts made by the European dosimetry group represent an international mobilization to expand knowledge about the risks and environmental parameters to be considered. The group has approximately 600 researchers who work with ionizing radiation dosimetry and develop research projects, educational and training actions, in addition to making interlaboratory comparisons [13]. Noteworthy is the fact that the actions developed need to consider and at the same time enable safety for health workers, regardless of whether they are responsible for operating ionizing radiation sources or if the exposure of health workers occurs in environments shared with multidisciplinary teams, as in the ICU. Thus, the perception of the importance of continuing educational actions, but involving all possible exposed workers, will enable the adoption of safe practices and the use of equipment that minimizes the exposure of health professionals.

\section{Conclusion}

The research on radioprotection and dimensioning of the effects of ionizing radiation in health professionals needs to be expanded and its proportionality inverted. In view of the applications of this type of radiation during work activity, professionals may be exposed to risks not yet dimensioned in Brazil. Educational actions play an important role in prevention; however, there are still no data on changing attitudes after long periods without training. It is believed that international efforts need to be made and directed towards investigations that promote changing attitudes, strengthening legislation, greater knowledge about the dimensioning of the effects and area of radiation in hospital environments. The use of radiation allowed the industrialization of medicine, promoting categories of professionals and expanding the possibilities of diagnosis and treatment. Actions to promote occupational health can collaborate with changes in behavior, suggesting safer and more conscious behaviors. Such actions can reduce the unnecessary exposure of the team, reducing risks, absenteeism and pathologies associated with the use of ionizing radiation.

\section{Conflict of Interest}

Thera are any economic or conflict of interest. 


\section{References}

1. Fundação Oswaldo Cruz (2020) Radiation.

2. (2012) International Agency for Research on Cancer, IARC Monograph on the identification of carcinogenic hazards to human. Radiation, Volume 100D

3. FUENTE M, Stephanie Long, David Fenton L, Chi Hung, Jamie Goggins, et al. (2020) Review of recent radon research in Ireland, OPTI-SDS project and its impact on the National Radon Control Strategy. Applied Radiation and Isotopes 163.

4. (2016) World and Health Organization WHO, Ionizing radiation, health effects and protective measures.

5. (2020) Ionizing Radiation. Ministério da Saúde.

6. (2019) Ionizing radiation. National Institute of Cancer.

7. (2014) Basic Diretrizes Of Proteção Radiológica. CNEN.

8. LEYTON F, Lucia Canevaro, Adriano Dourado, Helio Castello, Alexandre Bacelar, et al. (2014) Radiation risks and the importance of radiological

ISSN: 2574-1241

DOI: 10.26717/BJSTR.2020.30.004920

Katia Soares da Poça. Biomed J Sci \& Tech Res

(C) This work is licensed under Creative

Submission Link: https://biomedres.us/submit-manuscript.php protection in interventional cardiology: a systematic review. Brazilian Journal of Invasive Cardiology 22(1): 87-98.

9. DE Cássia Flôr, R Kirchhof, A L Cardoso (2006) An educational practice to raise awareness about exposure to ionizing radiation with health professionals. 59(3): 274-278.

10. Tang F R, Loganovsky K (2018) Low dose or low dose rate ionizing radiation-induced health effect in the human. Journal of Environmental Radioactivity 192: 32-47.

11. (2005) Regulatory Standard 32 - Nr 32 Safety And Health At Work In Health Services.

12. Hankin R A, Jones S P (2020) The impact of educational interventions on clinicians' knowledge of radiation protection: An integrative review. Radiography 26(3): 179-185.

13. Rühm W, E Ainsbury, B Breustedt M Caresana P Gilvin, et al. (2019) The European Radiation Dosimetry Group - Review of Recent Scientific Achievements. Radiation Physics and Chemistry 168.

BIOMEDICAL
RESEARCHES $\quad \begin{aligned} & \text { Assets of Publishing with us } \\ & \text { - Immediate, unrestricted online access }\end{aligned}$

